\title{
LVSI Pozitif ve Grade 1-2 Tümörü Olan Evre IA ve Evre IB Endometrial Kanser Hastalarının Karşılaştırılması: 2016 Yılında ESMO-ESGO ESTRO Tarafından Tanımlanmış Olan Yüksek-Orta Risk Grubunun Analizi
}

\author{
Comparison of Stage IA and Stage IB Endometrial Cancerpatients who were Unequivocally LVSI Positive \\ and Grade 1-2: Analysis of High Intermediate Risk Group which was \\ Currently Defined by ESMO-ESGO-ESTRO Consensus in 2016
}

\begin{abstract}
Koray ASLAN ${ }^{1}$, İbrahim YALÇIN ${ }^{1}$, Hanifi ȘAHİN ${ }^{1}$, Mehmet Mutlu MEYDANLI ${ }^{1}$
1. Jinekolojik Onkoloji Cerrahisi Bölümü, Dr. Zekai Tahir Burak Kadın Sağlı̆̆ı Eğitim ve Araştırma Hastanesi, Tip Fakültesi, Sağllk Bilimleri Üniversitesi, Ankara, Türkiye
\end{abstract}

Çalışma, sözel bildiri olarak “12thTurkish - German Gynecologic Congress. Kyrenia, Cyprus. 27-30.04.2018” kongresinde sunulmuştur

\section{$\ddot{O} Z E T$}

Amaç: Endometrial kanserinde, nüks riski olan hastaları belirlemek ve adjuvan tedavi kullanımına rehberlik etmek amacı ile klinik-patolojik prognostik faktörler kullanılarak risk grupları oluşturulmuştur. Güncel yayınlanan rehberde yeni bir risk grubu tanımlanmuş olup evre I endometrioid karsinom, grade 1-2 hastalik ve LVSI pozitif olan olgular myometrial invazyon derinliğinden bağımsız olarak "orta-yüksek" riskli olarak slnıflandirılmıştır. Bu retrospektif çalışmanın amacı evre IA yüksek-orta riskli endometrial kanserli kadınların prognozların evre IB Yüksek-orta riskli endometrial kanserli kadınlarla karşılaştırmaktır.

Gereçler ve Yöntem: Retrospektif tek merkezli olarak planlanan çalışmada, 2008-2018 tarihleri arasında endometrium kanseri tanist ile primer tedavi olarak cerrahi ile tedavi edilen tüm hastalar incelendi. Calıșaya evre I endometroid tip endometrium kanseri olup, LVSI pozitif ve grade 1-2 tümörü olan toplam 46 olgu dahil edildi. Calısmaya dahil edilen 17 olgu evre IA ve 29 olgu evre IB olmak üzere gruplandırlld. Olgular için să̆ kalım analizleri Kaplan Meier metodu kullanılarak hesaplandl.

Bulgular: 5-ylllı DFS oranı evre IA olgular için \%94, 1 ve evre IB olgular için \% 82,3 ( $p=0.951), 5$ - yıllık OS oranı evre IA olgular için \% 94,1 ve evre IB olgular için \% $89(p=0.811)$ olarak belirlenmiştir.

Sonuç: Evre IA, grade 1-2 ve LVSI pozitif endometrial kanserli olgular ve Evre IB, grade 1-2 ve LVSI pozitif endometrial kanserli olguların DFS ve OS oranlarının benzer olduğu görülmüştür.

Anahtar Kelimeler: endometrium kanseri, LVSI, săg kalım

\section{ABSTRACT}

Objective: The patients with endometrial cancer have been devised based on clinic-pathological prognostic factors to identify patients at the risk of recurrence and to guide adjuvant therapy use. According to contemporary guidelines, a new risk subgroup has been declared. Regardless of depth of invasion, all patients who were unequivocally LVSI positive and grade 1-2 defined as High-intermediate risk group. The purpose of this retrospective study was to compare the prognoses of women with stage IA high-intermediate endometrial cancer to those women of stage IB High-intermediate endometrial cancer.

\section{İletissim}

Sorumlu Yazar: Koray ASLAN, Jink. Onk. Cerrahisi Yandal Asistan Adres: Zekai Tahir Burak Kadın Sağlığı Eğitim ve Araştırma Hastanesi, Talatpaşa Bulvarı, 06230 Altındağ, Ankara, Türkiye Tel: +90 (505) 7107765

E-Posta: aslan_koray@yahoo.com

Makale Geliș: 02.07.2019

Makale Kabul: 29.08.2019

DOI: http://dx.doi.org/10.16948/zktipb.585846
Material and Methods: A single center, retrospective department database review was performed to identify patients with endometrial Cancer. A total of 46 women with Stage I endometrial cancer who were unequivocally LVSI positive and grade 1-2 between 2008 and 2018 were included in this retrospective study. Seventeen (37\%) were classified as Stage IA and 29 $(63 \%)$ as Stage IB. Kaplan-Meier method was used to generate survival data.

Results: The 5-year disease-free survival (DFS) rate was $94.1 \%$ versus $82.3 \%(p=0.95)$ and 5 -year overall survival (OS) was $94.1 \%$ versus $89 \%(p=0.81)$ for stage IA and stage $I B$, respectively.

Conclusion: DFS and OS rates of patients with Stage IA, grade 1-2 and LVSI positive endometrial cancer and Stage IB, grade 1-2 and LVSI positive endometrial cancer seem to be similar.

Keywords: endometrial cancer, LVSI, survival

\section{GÍRIS}

Endometrium kanseri gelişmiş ülkelerde en sık görülen jinekolojik malignite olup, en sık saptanan alt tipi endometroid tip endometrial kanserdir [1] [2]. Hastaların çoğu erken evrede tanı alırlar ve erken evre hastalıkta 5 yıllık sağ kalım \% 80'i aşmaktadır [3]. Endometrium kanseri öncelikle cerrahi olarak tedavi edilir. International Federation of Obstetricians and Gynecologists (FIGO) önerilerine göre evreleme cerrahisi histerektomi, salfingo ooferektomi ile pelvik ve paraaortik lenf nodlarının değerlendirilmesini kapsar. Hastalığın evresi, tümörün grade, histolojik tipi ve myometrial invazyon derinliği FIGO tarafından iyi tanımlanmış prognostik faktörlerdir [4]. Bu kliniko-patolojik faktörler hem hastalığın rekürrens riskinin öngörülmesinde hem de adjuvan tedavinin belirlenmesinde kullanılır.

Yapılan çalışmalarda evre I endometroid tip endometrium kanseri için hasta yaşının > 60 olmas1, myometrial invazyon derinliğinin \% 50 den fazla olması ve grade 3 hastalık olması rekürrens için risk faktörleri olarak tanımlanmıştır [5] [6]. Bu risk faktörlerinden bir tanesine sahip hastalar düşük riskli, iki tanesine sahip hastalar orta riskli ve üç tanesine sahip hastalar ise yüksek riskli olarak kategorize edilmiştir [7]. Hastaların adjuvan tedavilerinin bulundukları risk grubu göz önüne alınarak belirlenmesi hedeflenmiştir. Uterusta, esas tümörün dışında endotel ile çevrili kanalların içinde tümör hücrelerinin bulunması olarak tanımlanan lenfovasküler alan 
invazyonu (LVSI) ise bu sinıflandırmada risk faktörü olarak değerlendirilmemiştir [7]. Bununla birlikte, günümüzde LVSI varlığının lenf nodu tutulumu ile ilişkili olduğu bilinmektedir [8]. Ayrıca, LVSI saptanan endometrium kanserli olguların daha kötü prognoza sahip olduğu, daha düşük hastalıksız sağ kalım (DFS) oranı ve genel sağ kalım (OS) oranı ile ilişkili olduğu gösterilmiştir [9]. Zhang ve ark. evreleme cerrahisi yapilan 239 endometrial kanserli hastayı değerlendirmiş ve LVSI varlığını lokorejyonel nüks için bağımsız bir risk faktörü olarak tanımlamışlardır [10].

Erken evre endometrial kanserli hastalarda LVSI varlığının önemli bir prognostik faktör olduğunun bilinmesine karşın bu bilginin hastaların yararına nasıl kullanılacağ kadar fikir birliğine varılamamıştır. 2016 yılında, European Society of Gynaecological Oncology (ESGO) tarafindan yayınlanan rehberde yeni bir risk grubu tanımlanmıştır. Evre I endometrioid karsinom, grade 1-2 hastalik ve LVSI pozitif olan olgular myometrial invazyon derinliğinden bağımsız olarak "orta-yüksek" riskli olarak sınıflandırılmıştır. Bu hastalara lenfadenektomi sonuçları da göz önünde bulundurularak adjuvan tedavi olarak vajinal brakiterapi (VB) veya eksternal beam radyasyon tedavisi (EBRT) önerilmiştir [11].

$\mathrm{Bu}$ retrospektif çalışma ile ESGO tarafından yeni tanımlanan orta-yüksek risk grubunda (LVSI pozitif evre I endometrial kanserli olgularda) tümörün myometrial invazyon durumunun hastalığın prognozu üzerine önemini belirlemek hedeflenmiştir. Bu bağlamda, evre IA orta-yüksek riske sahip hastaların prognozları evre IB orta-yüksek risk grubundaki hastalarla karşılaştırılmıştır.

\section{GEREÇ ve YÖNTEM}

Dr. Zekai Tahir Burak Kadın Sağlığı Eğitim ve Araştırma Hastanesi'nde 2008-2018 tarihleri arasinda endometrium kanseri tanisı ile primer tedavi olarak cerrahisi uygulanan tüm olgular geriye dönük olarak incelendi. Çalışma için hastane etik kurulundan onay alındı (kabul tarihi: 28.9.2018, kabul no: 18). Operasyon öncesinde, çalışmaya dahil edilen tüm hastalardan, hastanın klinik verilerinin kurumumuz tarafindan kullanılmasina izin veren bilgilendirilmiş onam formu alındı.

Çalışmaya, FIGO 2009 kriterlerine göre evre I endometroid tip endometrium kanseri saptanıp, nihai patoloji raporuna göre grade $1 / 2$ ve LVSI pozitif tümörü olan toplam 46 olgu dahil edildi. Tibbi kayıtları eksik olan olgular ve eşlik eden başka bir primer malignitesi olan hastalar çalışma kapsamı dışında bırakıldı.

Tüm operasyonlar deneyimli jinekolojik onkoloji cerrahisi uzmanları tarafından yapıldı. Tüm hastalara öncelikle total histerektomi ve bilateral salfingo-oferektomi yapıldı. Pelvik ve paraaortik lenfadenektomi operasyona katılan cerrahın tercihine göre rutin olarak yapıld 1 veya histerektomi materyalinin frozen patoloji ile değerlendirilmesi sonucunda uygulandi. Tüm Patoloji spesmenleri jinekolojik patoloji alanında deneyimli patoloji uzmanları tarafından incelendi. Tümörün yapısal gra- de i FIGO kriterlerine göre belirlendi [12]. LVSI, primer tümörün dışında cerrahi spesimende herhangi bir endotel ile çevrili kanalda tümör saptanması olarak tanımlandı [13]. Hastaların evreleri FIGO 2009 önerileri doğrultusunda belirlendi. Adjuvan tedavi kararı güncel bilgiler doğrultusunda risk faktörleri dikkate alınarak verildi.

Hastane kayıtları gözden geçirilerek hastaların operasyon sırasındaki yaşı, tümör boyutu, tümör grade i, çıkarılan toplam lenf nodu sayısı, nüks durumu, median takip süresi ve aldıkları adjuvan tedavi gibi klinik, patolojik ve demografik verileri kayıt edildi. OS, cerrahi tedavi ile herhangi bir sebepten ölüm veya takip dışı kaldıysa son takip tarihi arasından geçen süre olarak hesapland1. DFS ise cerrahi tedavi ile nüks ve ölüm durumlarından önce gerçekleşenin tarihi veya son takip tarihi arasındaki süre olarak hesaplandi. Hastalar FIGO evre IA ve evre IB olmak üzere iki gruba ayrildı ve gruplar belirtilen değişkenler açısından karşılaştırıldı.

İstatistiksel analiz SPSS versiyon 23 (IBM Corp., Armonk, NY, USA) kullanilarak yapildı. Sayısal değişkenler dağılım durumuna göre (Kolmogorov-Smirnov/Shapiro-Wilk) ortanca ile tanımland 1 ve Mann Whitney U testi kullanılarak karş1laştırıldı. Kategorik değişkenler ise yüzde ve oran kullanılarak gösterildi ve Chi-kare testi ile karşılaştırıldı. Olgular için sağ kalım analizleri Kaplan Meier metodu ve log-rank testi kullanılarak hesapland. Tüm karşılaştırmalar için $\mathrm{p}<0,05$ değeri anlamlı olarak kabul edildi.

\section{BULGULAR}

Hastanemizde 2008 -2018 y1lları arasında endometrial kanser tanisı ile opere edilen ve nihai patoloji sonucuna göre FIGO evre I, LVSI pozitif ve grade $1 / 2$ hastalığa sahip 46 olgu çalışmaya dahil edildi. Hastalar FIGO evrelerine göre evre IA $(n=17)$ ve evre IB $(n=29)$ olmak üzere iki gruba ayrild1.

Evre IA olguların median yaşı 55 (40-69 y11) ve evre IB olguların median yaşı 62 (49-85) olarak saptanmıştır. Evre IB hastalığa sahip kadınların istatiksel olarak anlamlı olacak şekilde daha yaşlı olduğu gösterilmiştir $(\mathrm{p}=0,004)$. Evre IA olgular ve evre IB olgular tümör boyutu $(\mathrm{p}=0,397)$, tümör grade $(\mathrm{p}=0,555)$, lenfadenektomi yapilan hasta say1s1 $(p=0,174)$, çıkarlan toplam lenf nodu sayıs1 $(p=0,614)$ açısından benzer bulunmuştur. Adjuvant radyoterapi, evre IB hastalara evre IA hastalara göre istatiksel olarak anlamlı olacak şekilde daha sık uygulanmıştır (sırayla, \% 41,2 ve \% 79,3; $p=0,012$ ).

Ortalama takip süresi evre IA olgular için 43.5 ay (1-122 ay) ve evre IB olgular için 37 ay (4-111 ay) olarak hesaplanmıştır. Her iki grubun ortalama takip süreleri açısından benzer olduğu gösterilmiştir $(\mathrm{p}=0,135)$. Beş y1llık DFS oranı evre IA olgular için $\% 94,1$ ve evre IB olgular için \% 82,3 olarak belirlenmiştir.

Gruplar arasında DFS açısından istatiksel olarak anlamlı fark saptanmamıştır $(\mathrm{p}=0.951)$. Beş y1ll1k OS oranı evre IA olgular için \% 94,1 ve evre IB olgular için \% 89 olup, grupların OS açısından benzer olduğu gösterilmiştir ( $\mathrm{p}=0.811$ ). 


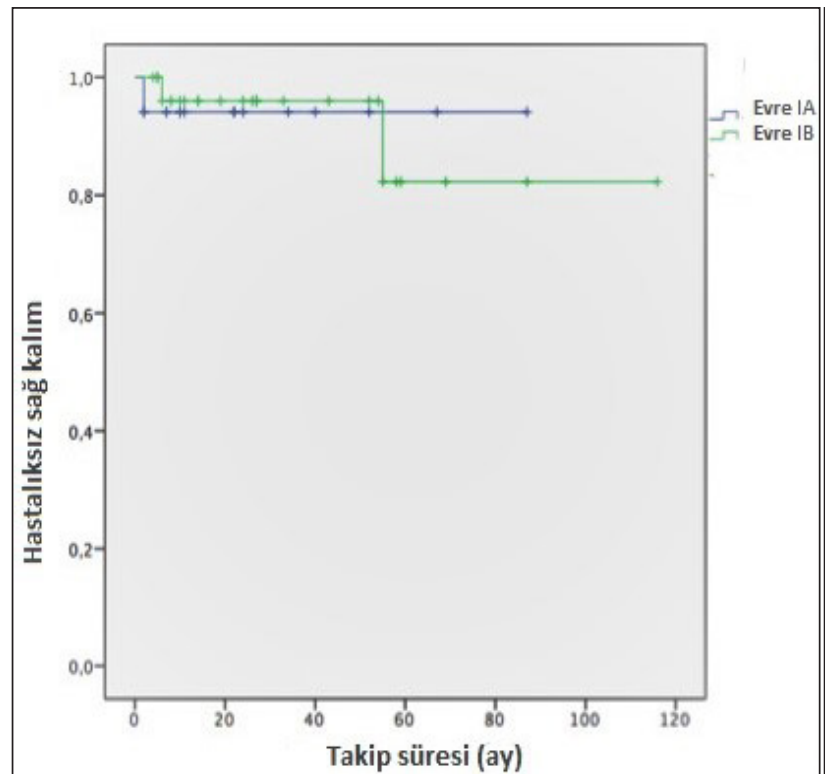

Şekil 1: LVSI poziti ve grade $1 / 2$ tümöre sahip endometrium kanserli hastaların hastalıksız sağ kalımlarının karşılaştırılması.

Tablo 1: Endometrium kanseri LVSI pozitif, grade 1/2 hastaların demografik ve klinikopatolojik özellikleri.

\begin{tabular}{|c|c|c|c|}
\hline & Evre IA (n=17) & Evre IB $(n=29)$ & $\mathrm{p}$ \\
\hline Yaş, yıl (median) & $55(40-69)$ & $62(49-85)$ & 0,004 \\
\hline Tümör boyutu & & & \\
\hline $\begin{array}{l}\leq 2 \mathrm{~cm} \\
>2 \mathrm{~cm}\end{array}$ & $\begin{array}{l}4(23.5 \%) \\
13(76.5 \%)\end{array}$ & $\begin{array}{l}3(10.3 \%) \\
26(89.7 \%)\end{array}$ & 0,397 \\
\hline Grade (n) & & & \\
\hline $\begin{array}{l}1 \\
2\end{array}$ & $\begin{array}{l}6(35.3 \%) \\
11(64.7 \%)\end{array}$ & $\begin{array}{l}13(44.8 \%) \\
16(55.2 \%)\end{array}$ & 0,555 \\
\hline Lenfadenektomi, $\mathrm{n}$ & & & \\
\hline $\begin{array}{l}\text { Yok } \\
\text { Var }\end{array}$ & $\begin{array}{l}4(23.5 \%) \\
13(76.5 \%)\end{array}$ & $\begin{array}{l}2(6.7 \%) \\
27(93.1 \%)\end{array}$ & 0,174 \\
\hline $\begin{array}{l}\text { Çıkarılan Lenf nodu } \\
\text { sayısı, median (range) }\end{array}$ & $51(29-67)$ & $48(22-119)$ & 0,614 \\
\hline Nüks sayısı, n & & & \\
\hline $\begin{array}{l}\text { Yok } \\
\text { Var }\end{array}$ & $\begin{array}{l}11(15.7 \%) \\
59(84.3 \%)\end{array}$ & $\begin{array}{l}47(33.6 \%) \\
93(66.4 \%)\end{array}$ & 0,008 \\
\hline Median takip süresi, ay & $43.5(1-122)$ & $37(4-111)$ & 0,135 \\
\hline $\begin{array}{c}\text { Adjuvan radyoterapi } \\
\qquad \begin{array}{c}\text { Yok } \\
\text { Var }\end{array}\end{array}$ & $\begin{array}{l}10(58,8 \%) \\
7(41,2)\end{array}$ & $\begin{array}{l}6(20,3) \\
23(79,3)\end{array}$ & 0,012 \\
\hline
\end{tabular}

Koyu karakterler istatiksel olarak anlamlı farkı göstermektedir. n: say1

Tablo 2: LVSI poziti ve grade 1/2 tümöre sahip hastaların sağ kalım özellikleri.

\begin{tabular}{|l|l|l|l|}
\hline & Evre IA & Evre IB & $\mathrm{p}$ \\
\hline 5-y1llk DFS, \% & 94,1 & 82,3 & 0,951 \\
\hline 5-y1llk OS, \% & 94,1 & 89,0 & 0,811 \\
\hline
\end{tabular}

DFS: hastalıksız sağ kalım, OS: genel sağkalım

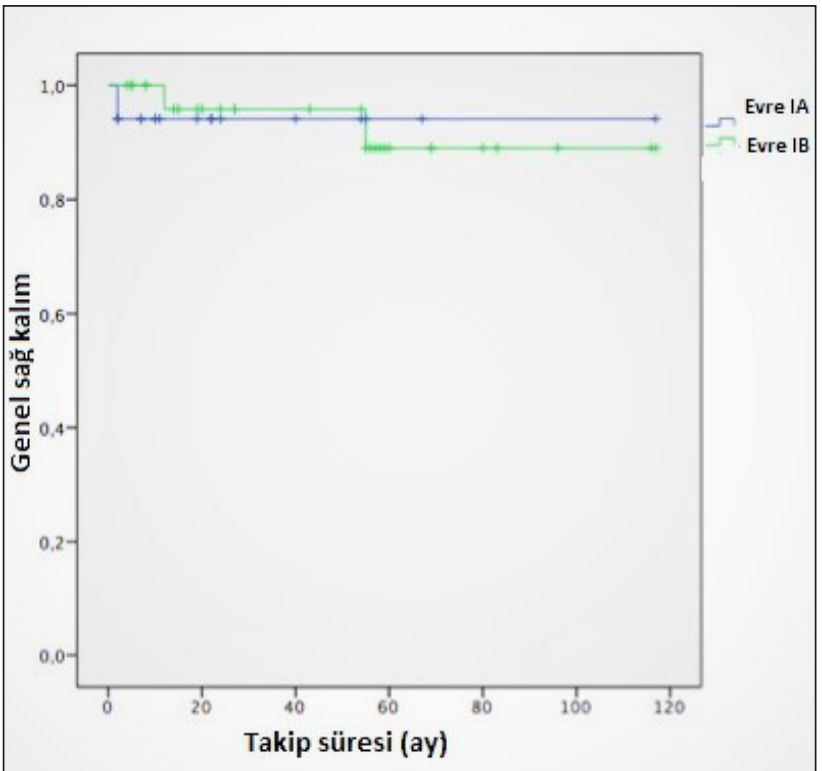

Sekil 2: LVSI poziti ve grade 1/2 tümöre sahip endometrium kanserl hastaların genel sağ kalımlarının karşılaştırılması.

\section{TARTIȘMA}

Bu çalışmada, nihai patoloji sonucuna göre evre I endometroid tip endometrium kanseri tespit edip, LVSI pozitif ve grade $1 / 2$ tümörü olan hastalarda, myometrial invazyon derinliğinin hastalığın prognozunu üzerindeki etkisi değerlendirilmiştir. Çalışma sonucunda, evre IA olgular ile evre IB olguların benzer OS ve DFS oranına sahip olduğu gösterilmiştir.

Çalışmamızın temel kısıtlılı̆g retrospektif olarak tasarlanması ve olgu sayısının az olmasidır. Evre I endometrium kanserinde nüks veya ölüm sıklığ1nın az olduğu göz önünde bulundurulduğunda evre IA hastalığa sahip olgularla evre IB hastalığa sahip olgular arasındaki sağ kalım farkını daha geniş hasta grubu ile değerlendirmek faydalı olabilir. Çalışmamızın diğer kısitlllığ 1 ise patoloji spesimenlerinin merkezi olarak tekrar değerlendirilmemesidir. Buna karşın, tüm hastaların deneyimli jinekolojik onkolojisi cerrahları tarafindan opere edilmesi ve tüm patoloji sonuçlarının jinekolojik patoloji alanında deneyimli patoloji uzmanları tarafından değerlendirilmesi çalıșmamızın güçlü yanlarıdır.

Endometrium kanserinde LVSI varlığının lenf nodu tutulumu ile ilişsili olduğu bilinmektedir [14]. Ayrica, erken evre endometrium kanserinde LVSI pozitif olguların daha s1k nüks ettiği ve hastalığın daha kötü prognoza sahip olduğu gösterilmiştir [15]. Fakat endometrium kanserli hastalarda adjuvan tedavi planlanırken LVSI pozitifliğinin oynaması gereken rol konusunda uzun süre fikir birliğine varılamamıștır. ESGO tarafinda 2016 yılında yayinlanan rehberde, LVSI operasyon sonrasinda adjuvan tedavinin belirlenmesinde kullanılan risk faktörlerinden biri olarak tanımlanmıştır. Evre I, grade $1 / 2$ olgular, myometrial invazyon derinliği dikkate alınmaksızın "orta-yüksek" riskli grup olarak belirlenmiştir. Bu öneriler sonucunda, LVSI pozitif evre IA ve evre IB olguların nüks açısından benzer riske sahip oldukları öne sürülmüștür. Buna karşın, hem PORTEC-1 çalışmasında hem de GOG 99 çalışmasında myometrial invazyon derinliğinin 
$>\% 50$ olmas1 endometrium kanserinde daha kötü prognozla ilişkili bulunmuştur [16] [17].

Han ve ark. tarafindan 2017 yılinda yapilan 521 kadının dahil edildiği çalışmada, evre I endometroid tip endometrium kanserinde prognostik faktörlerin belirlenmesi hedeflenmiștir. Multivariate analiz sonucunda myometrial invazyon derinliği ( Hazard ratio (HR) 9,803; Confidence interval (CI) 2,003-47,968) ve tümörün grade (HR 2,764; CI 0,423-18,054) DFS ile ilişskili bulunmuștur. LVSI pozitifliği ise DFS için risk faktörü olarak gösterilememiştir [18]. Tamamına lenfadenektomi yapılmış, evre I endometrium kanserli 229 hastanın (lenf nodu negatif) değerlendirildiği çalışmada Mariani ve ark. çeşitli histopatolojik faktörlerin hastalığın prognozu ile olan ilişkisini değerlendirmişlerdir. Univariate analizde myometrial invazyon $\geq \% 66$, non- endometroid histoloji, tümör çap $1>2$ $\mathrm{cm}$ hastalığa özgü sağ kalımla ilişkili bulunmuştur $(\mathrm{DRS})(\mathrm{p}<0,05)$. LVSI pozitifliği ile DRS arasında anlamlı ilişki saptanmamıştır $(\mathrm{p}=0,06)$. Multivariate analizde ise yalnızca myometrial invazyon $\geq \%$ 66 daha kötü DRS için bağımsız prognostik faktör olarak tanımlanmıștır (Relative risk 12, 44; \%95 CI $3,48-44,42, p=0,001$ ) [19]. Buna karşın, Guntupalli ve ark. 511 endometroid tip endometrial kanserli olguyu değerlendirdikleri çalışmalarında; LVSI (HR 2,14; CI 1,53-2,88), derin myometrial invazyon (HR 1,47; CI 1,09-1,97), hastanın yaş1 (HR 1.033; CI 1.02-1.05) ve tümörün grade (HR 1.59; CI 1.331.91) DFS için bağımsız risk faktörü olarak tespit edilmiştir [8].

Dos Reis ve arkadaşları, evre IA grade 1-2 hastalığa sahip 240 endometrial kanserli hastayı dahil ettikleri çalışmalarında LVSI durumunun hastalığın prognozu üzerine olan etkisini değerlendirmişlerdir. Olguların 40'1nda (\% 16,7) LVSI pozitif olduğu bildirilmiştir. Çalışma sonucunda LVSI pozitifliği anlamlı şekilde daha kötü DFS $(p=0,002)$ ve OS $(p=0,013)$ ile ilişkili bulunmuştur [20]. 2014 yılında yapılan çalışmada Aristizabal ve ark. evre I endometrium kanserinin LVSI negatif ve LVSI pozitif olmak üzere iki grubu ayrılması gerektiğini öne sürmüştür. Çalışmaya 508 evre I endomerial kanserli olgu dahil edilmiş ve hastalar hem FIGO önerileri dikkate alınarak tümörün myometrial invazyon durumuna göre ( myometrial invazyon derinliğ $i<\% 50$ veya $\geq \% 50$ ) hem de LVSI durumlarına göre (LVSI pozitif ve LVSI negatif) gruplandırılmıştır. Hangi yaklaşımın 5-yıllık OS oranını daha iyi ön gördüğü belirlenmeye çalışılmıştır. Sonuç olarak LVSI pozitif ve LVSI negatif grupları arasındaki 5-yıllık OS fark 1 istatiksel olarak anlamlı iken (sırayla, $\% 81$ ve $\% 97, \mathrm{p}=0,009)$, myometrial invazyon $\geq \% 50$ ve $<\% 50$ grupları arasında 5 yıllık OS açısından anlamlı fark saptanmamıştır (sırayla, \% 87 ve \% 96, $\mathrm{p}=0,09)$. Aynı çalışmada 5 yıllık OS oranı; LVSI negatif ve myometrial invazyon derinliği $<\% 50$ olan olgular için \% 98, LVSI negatif ve myometrial invazyon derinliği $\geq \% 50$ olan olgular için $\% 95$ ve myometrial invazyon derinliğinden bağımsız olarak LVSI pozitif olgular için \% 81 olarak hesaplanmıştır $(\mathrm{p}=0,03)$. Bu çalışma sonucunda, evre I endometrium kanserli olgularda LVSI durumunun FIGO evre IA veya IB olmasına göre hastalığın prognozunu ön görme konusunda daha değerli olduğu yorumunda bulunulmuştur [21]. Bizim çalışmamızda ise LVSI pozitif endometroid tip endometrium kanserli olgularda evre IA ve evre IB hastalık arasinda DFS (s1rasiyla, $\% 94,1$ ve $\% 82,3 ; p=0,95$ ) ve OS (sirasiyla, 94,1 ve $89 ; \mathrm{p}=0,81$ ) açısından istatiksel anlamlı fark saptanmamıştır. Olgu sayısı az olmasına karşın bulgular evre I hastalarda risk gruplarının LVSI temelli oluşturulmasını desteklemektedir.

Sonuç olarak evre IA, grade 1/2, LVSI pozitif endometrium kanserli olgular ve evre IB, grade 1-2, LVSI pozitif endometrial kanserli olguların benzer DFS oranı ve OS oranına sahip olduğu gösterilmiştir. Bu bulgular LVSI pozitif ve evre I hastalıkta myometrial invazyon derinliğinin prognostik değerinin sınırlı olduğu şeklinde yorumlanmaktadır. Bununla birlikte konuyla ilgili daha geniş olgu sayısına sahip çalışmalara ihtiyaç vardır.

\section{KAYNAKLAR}

1. Siegel RL, Miller KD, Jemal A. Cancer statistics, 2018. CA Cancer J Clin 2018; 68:7-30.

2. Amant F, Moerman P, Neven P, Timmerman D, Van Limbergen E, Vergote I. Endometrial cancer. Lancet 2005; 366:491505 .

3. Sorosky JI. Endometrial Cancer. Obstet Gynecol 2012; 120:383-397.

4. Pecorelli S. Revised FIGO staging for carcinoma of the vulva, cervix, and endometrium. Int J Gynaecol Obstet 2009; 105:103-4.

5. Keys HM, Roberts JA, Brunetto VL, Zaino RJ, Spirtos NM, Bloss JD, et al. A phase III trial of surgery with or without adjunctive external pelvic radiation therapy in intermediate risk endometrial adenocarcinoma: a Gynecologic Oncology Group study. Gynecol Oncol 2004; 92:744-751.

6. Nout RA, van de Poll-Franse L V, Lybeert MLM, Wárlám-Rodenhuis CC, Jobsen JJ, Mens JWM, et al. Long-Term Outcome and Quality of Life of Patients With Endometrial Carcinoma Treated With or Without Pelvic Radiotherapy in the Post Operative Radiation Therapy in Endometrial Carcinoma 1 (PORTEC-1) Trial. J Clin Oncol 2011; 29:1692-1700

7. Sadozye AH, Harrand RL, Reed NS. Lymphovascular Space Invasion as a Risk Factor in Early Endometrial Cancer. Curr Oncol Rep 2016; 18:24.

8. Guntupalli SR, Zighelboim I, Kizer NT, Zhang Q, Powell $M A$, Thaker PH, et al. Lymphovascular space invasion is an independent risk factor for nodal disease and poor outcomes in endometrioid endometrial cancer. Gynecol Oncol 2012; 124:31-35.

9. Weinberg LE, Kunos CA, Zanotti KM. Lymphovascular Space Invasion (LVSI) Is an Isolated Poor Prognostic Factor for Recurrence and Survival Among Women With Intermediate- to High-Risk Early-Stage Endometrioid Endometrial Cancer. Int J Gynecol Cancer 2013; 23:1438-1445.

10. Zhang $C$, Wang $C$, Feng $W$. Clinicopathological risk factors for pelvic lymph node metastasis in clinical early-stage endometrioid endometrial adenocarcinoma. Int J Gynecol Cancer 2012; 22:1373-7. 
11. Colombo N, Creutzberg C, Amant F, Bosse T, González-Martín A, Ledermann J,et al. ESMO-ESGO-ESTRO Consensus Conference on Endometrial Cancer: diagnosis, treatment and follow-up. Ann Oncol 2016; 27:16-41.

12. Shepherd JH. Revised FIGO staging for gynaecological cancer. Br J Obstet Gynaecol 1989; 96:889-92.

13. Keys HM, Roberts JA, Brunetto VL, Zaino RJ, Spirtos NM, Bloss JD, et al. A phase III trial ofsurgery with or without adjunctive external pelvic radiationtherapy in intermediate risk endometrial adenocarcinoma: Agynecologic oncology group study. Gynecol Oncol 2004; 92:744-751.

14. Cohn DE, Horowitz NS, Mutch DG, Kim SM, Manolitsas T, Fowler JM. Should the presence of lymphvascular space involvement be used to assign patients to adjuvant therapy following hysterectomy for unstaged endometrial cancer? Gynecol Oncol 2002; 87:243-6.

15. Loizzi V, Cormio G, Lorusso M, Latorre D, Falagario M, Demitri $P$, et al.The impact of lymph vascular space invasion on recurrence and survival in patients with early stage endometrial cancer. Eur J Cancer Care (Engl) 2014; 23:380-384.

16. Creutzberg CL, Nout RA, Lybeert MLM, Wárlám-Rodenhuis CC, Jobsen JJ, Mens J-WM, et al. PORTEC Study Group. Fifteen-Year Radiotherapy Outcomes of the Randomized PORTEC-1 Trial for Endometrial Carcinoma. Int J Radiat Oncol 2011; 81:e631-e638.

17. Keys HM, Roberts JA, Brunetto VL, Zaino RJ, Spirtos NM, Bloss JD, et al. Gynecologic Oncology Group. A phase III trial of surgery with or without adjunctive external pelvic radiation therapy in intermediate risk endometrial adenocarcinoma: a Gynecologic Oncology Group study. Gynecol Oncol 2004; 92:744-751.

18. Han KH, Kim HS, Lee M, Chung HH, Song YS. Prognostic factors for tumor recurrence in endometrioid endometrial cancer stages IA and IB. Medicine (Baltimore) 2017; 96:e6976.

19. Mariani A, Webb MJ, Keeney GL, Lesnick TG, Podratz KC. Surgical Stage I Endometrial Cancer: Predictors of Distant Failure and Death. Gynecol Oncol 2002; 87:274-280.

20. dos Reis R, Burzawa JK, Tsunoda AT, Hosaka M, Frumovitz $M$, Westin SN, et al. Lymphovascular Space Invasion Portends Poor Prognosis in Low-Risk Endometrial Cancer. Int J Gynecol Cancer 2015; 25:1292-9.

21. Aristizabal P, Graesslin O, Barranger E, Clavel-Chapelon $F$, Haddad B, Luton D, et al. A suggested modification to FIGO stage I endometrial cancer. Gynecol Oncol 2014; 133:192-196. 